\title{
Prevención primaria en fibrilación auricular
}

\section{Primary prevention in atrial fibrillation}

\author{
Gustavo A. Montero-Rincón ${ }^{\mathrm{a}, \mathrm{b}}$ \\ a Servicio de Electrofisiología Clínica Rey David, Cali, Colombia \\ b Departamento de Cardiología, Servicio de Electrofisiología Clínica Amiga, Cali, Colombia
}

Recibido el 3 de octubre de 2016; aceptado el 6 de octubre de 2016

Disponible en Internet el 4 de noviembre de 2016

\section{Resumen}

Dada la alta incidencia y prevalencia de la fibrilación auricular, es preciso revisar las recomendaciones en la prevención primaria de esta común arritmia en términos de nivel de evidencia y consistencia de la misma en los diferentes escenarios: para ello se realizó una búsqueda de estudios clínicos aleatorizados, así como se pusieron en contexto las recomendaciones de las Guías Clínicas.

Las medidas de prevención primaria son de vital importancia en cualquier proceso patológico. La fibrilación auricular ofrece dificultad especial dado que comparte factores de riesgo para otras enfermedades cardiovasculares, las cuales a su vez en sí mismas son factores de riesgo para esta. Los programas de manejo del riesgo enfocados en el control del peso, la promoción de hábitos saludables, la estimación individual del riesgo, el manejo de los factores de riesgo clásicos así como del síndrome de apnea/hipopnea del sueño, son herramientas prometedoras para la prevención primaria de la fibrilación auricular.

\section{Introducción}

La fibrilación auricular se demostró por primera en los electrocardiogramas hace más de 100 años, reconociéndose desde entonces como uno de los mayores problemas

Correo electrónico: gmontero99@hotmail.com de salud global. Se considera que más del $1 \%$ de la población en Australia, Europa y Estados Unidos está afectada por esta arritmia. Sin embargo, la prevalencia real puede desconocerse o subestimarse dado que muchos pacientes con fibrilación auricular permanecen no diagnosticados ${ }^{1}$. Se considera entonces como una epidemia de rápido crecimiento, de la que se proyecta que la prevalencia aumentará desde 5,2 millones en 2010 a 12,1 millones en 2030 debido especialmente al envejecimiento de la población. La fibrilación auricular se asocia con un incremento en mortalidad, morbilidad y alto costo de atención en salud.

Sin ser una sorpresa, muchos factores de riesgo modificables relacionados con enfermedades ateroscleróticas cardiovasculares han sido relacionados con la aparición de fibrilación auricular. Sin embargo, aunque las últimas Guías Prácticas de fibrilación auricular mencionan estos factores de riesgo, no hacen recomendaciones con algún nivel de evidencia en dieta, ejercicio o cambios en el estilo de vida para prevención primaria y secundaria de fibrilación auricular.

En 2013, la Sociedad de Ritmo Cardiaco (HRS, su sigla en inglés) reconoce "avances en los esfuerzos para la prevención de fibrilación auricular mediante enfoque en la modificación del riesgo" como un objetivo clave de investigación. Debe igualmente considerarse que existen otros factores de riesgo para fibrilación auricular como lo son las enfermedades valvulares, la enfermedad tiroidea y la enfermedad pulmonar obstructiva crónica, los cuales no son factores de riesgo clásicos ateroscleróticos pero tienen implicaciones para las estrategias de manejo. 
Históricamente, los aspectos de prevención primaria en fibrilación auricular han sido materia de desarrollo y decepción, probablemente obedeciendo a la complejidad de factores interrelacionados, desde el punto de vista fisiopatológico, con la aparición de la fibrilación auricular, algunas veces modificables y otras difícilmente intervenibles. A pesar del uso de medicamentos que intervienen el sistema renina-angiotensina-aldosterona (SRAA), hipolipemiantes como atorvastatina y ácidos grasos omega (propuestos como estrategias de prevención primaria cuando tengan indicación por otras causas), existen vacíos terapéuticos cuando se pretende intervenir de manera primaria la fibrilación auricular.

Se plantean otras estrategias de intervención en forma de programa de intervención de riesgo de fibrilación auricular, que comprende atacar los factores de riesgo clásicos cardiovasculares y otros más recientemente reconocidos como pivotes en la aparición de fibrilación auricular.

\section{Obesidad y pérdida de peso}

El aumento en la prevalencia de la obesidad ha sido claramente documentado en los Estados Unidos de América (EUA), donde se estima que un tercio de los adultos son obesos, así como globalmente ${ }^{2}$.

En los últimos cinco años, numerosos estudios establecen que la obesidad está íntimamente relacionada con la aparición de fibrilación auricular. El índice de masa corporal (IMC) forma parte de los modelos de predicción para el inicio de fibrilación auricular. Se ha demostrado, igualmente, que esta asociación es independiente de otras comorbilidades asociadas a la obesidad.

En el Estudio ARIC (Atheroesclerotic Risk in Communities) $(n=14.598)$ se atribuyó un $17 \%$ de riesgo de fibrilación auricular a la presencia de obesidad o sobrepeso ${ }^{3}$. Así mismo, se demostró mayor riesgo de desarrollar fibrilación auricular en los estudios WHS (Women's Health Study) con 5\% de riesgo por cada $\mathrm{kg} / \mathrm{m}^{2}$ de aumento en el IMC y en el WHI (Women's Health Initiative) con $12 \%$ de riesgo a igual magnitud de ascenso en el $\mathrm{IMC}^{4}$.

De manera interesante, en el WSH, se encontró que mayores niveles de actividad física atenuaron el nivel de riesgo ${ }^{5}$. Un índice de masa corporal $(I M C)>35 \mathrm{~kg} / \mathrm{m}^{2}$, también se relacionó con un riesgo 3,5 veces mayor de fibrilación auricular en mujeres jóvenes y saludables por demás ${ }^{6}$. En un metaanálisis reciente de 51 estudios con 626.603 pacientes, se encontró un 10-29\% riesgo relativo mayor de fibrilación auricular por cada $5 \mathrm{~kg} / \mathrm{m}^{2}$ de aumento en el IMC ya sea fibrilación auricular de novo o postoperatoria ${ }^{7}$.

Adicionalmente, medidas de adiposidad diferentes a la estimación del IMC, también se han asociado con mayor riesgo de fibrilación auricular. Un registro danés con 55.273 pacientes y 13,5 años de seguimiento, encontró que una masa corporal de grasa aumentada, medida con impedancia bioeléctrica, se relacionó con mayor incidencia de fibrilación auricular ${ }^{8}$.

Diversos mecanismos subyacen entre la asociación de fibrilación auricular y la obesidad. En un modelo animal de una dieta alta en calorías, la obesidad se asoció con dilatación y fibrosis de la aurícula izquierda, infiltración grasa e inflamatoria en la aurícula, así como cambios en las propiedades eléctricas, que llevan a tasas altas de fibrilación auricular espontánea o inducida.

En humanos, un alto valor de IMC se ha relacionado con dilatación de la aurícula izquierda ${ }^{9}$, lo cual a su vez se asocia con mayor riesgo de fibrilación auricular ${ }^{10}$.

El aumento del volumen de la grasa pericárdica se ha descrito en humanos obesos y se ha relacionado igualmente con la fibrilación auricular así como con la severidad y recurrencia después de ablación, lo cual ha sido independiente del valor de IMC y sugiere un efecto patológico local de la grasa pericárdica ${ }^{11}$. La obesidad también se ha asociado con aumento en el grosor de la grasa pericárdica, lo cual conlleva alteraciones electrofisiológicas y desbalance simpático-vagal en la aurícula izquierda ${ }^{12}$. Clínicamente, la grasa pericárdica se ha relacionado con fibrilación auricular ${ }^{13}$.

Finalmente, la obesidad se coliga a un estado de inflamación crónica, sistémica y de bajo grado, que desempeña un papel clave en la iniciación y perpetuación de la fibrilación auricular, favoreciéndose así la asociación entre obesidad y fibrilación auricular ${ }^{14}$.

Estudios clínicos recientes demuestran que la reducción de peso ejerce un rol importante en el contexto de un programa de modificación de factores de riesgo para el manejo de la fibrilación auricular.

Un estudio en un solo centro con 150 pacientes obesos con fibrilación auricular sintomática, aleatorizó a unos para manejo de sobrepeso y a otros para consejería de estilo de vida, ambos grupos en terapia común para fibrilación auricular. El programa de manejo de peso consistió en la prescripción de una rutina de ejercicio y una dieta hipocalórica, con una mejoría en el IMC desde 32,8 a $27,2 \mathrm{~kg} / \mathrm{m}^{2}$ en 15 meses vs. ningún cambio en el grupo control. A los 15 meses, el grupo de control de peso mediante programa, redujo la frecuencia de episodios de fibrilación auricular y la duración de los mismos y obtuvo menores puntajes en las pruebas de severidad de los síntomas ${ }^{15}$.

El estudio LEGACY-AF (Long Term Effect of Goal Directed Weight Management on Atrial Fibrillation Cohort: A 5 Year Follow Up Study) evaluó el impacto del programa de modificación de factores de riesgo y pérdida de peso en el manejo de la fibrilación auricular a mayor plazo y detectó que una pérdida de peso mayor al $10 \%$ estuvo asociada a una sobrevida libre de fibrilación auricular seis veces mayor (en pacientes con IMCD > 27). Igual efecto se ha encontrado en pacientes sometidos a ablación de fibrilación auricular ${ }^{16}$.

Por lo tanto, el nexo epidemiológico entre la obesidad y la fibrilación auricular está claro y hoy existe mayor comprensión de la fisiopatología que une las dos condiciones. Los datos recientes apoyan la importancia de la reducción del peso en la prevención y el manejo de la fibrilación auricular. Cabe anotar que los estudios mencionados no tomaron solamente la reducción de peso per se, sino un programa de ejercicio, dieta y modificación de otros factores existentes. Tal nexo entre pérdida de peso y fibrilación auricular es por tanto multifactorial, pues un programa de modificación de factores de riesgo con recomendación de reducción de peso, conlleva una mejoría en otras condiciones comórbidas que también están independientemente relacionadas con fibrilación auricular. Sin embargo, la pérdida de peso sí resulta en cambios estructurales en la aurícula apoyando la teoría al respecto ${ }^{17}$. En la actualidad, la evidencia clínica 
es suficiente para recomendar fuertemente la pérdida de peso tanto para la prevención como para el manejo de la fibrilación auricular.

\section{Ejercicio y entrenamiento}

Los beneficios del ejercicio rutinario están bien establecidos y recomendados en la literatura. Algunos estudios observacionales han demostrado mayor riesgo de fibrilación auricular en atletas jóvenes o en deportistas de alta resistencia ${ }^{17}$.

En el Estudio de Salud Cardiovascular (Cardiovascular Health Study), la incidencia de fibrilación auricular fue menor en aquellos individuos que practicaban ejercicios livianos a moderados que en aquellos que no hacen ninguna $\operatorname{actividad}^{18}$. No hubo diferencias en la aparición de fibrilación auricular entre personas que hacen ejercicio de alta intensidad y las que no hacen ejercicio. La incidencia también fue menor en los caminantes de largas distancias o a pasos rápidos que en los otros caminantes. Los beneficios del ejercicio han sido igualmente señalados en diferentes estudios de pacientes obesos y no obesos ${ }^{19}$.

Dados los demás beneficios cardiovasculares del ejercicio de manera rutinaria, es lógico recomendar el ejercicio moderado y regular como parte de la prevención de la fibrilación auricular y en el manejo de la misma.

\section{Hipertensión arterial}

Ha sido de manera consecutiva uno de los más fuertes predictores del desarrollo de fibrilación auricular ${ }^{20}$. Aún los rangos normales-altos de presión arterial se han relacionado con mayor riesgo de fibrilación auricular. El aumento de la postcarga conduce a remodelamiento estructural auricular y ventricular y consiguientes disfunción diastólica, dilatación auricular izquierda y fibrosis, lo cual a su vez lleva a fibrilación auricular ${ }^{21,22}$. No se ha demostrado una reducción consistente del riesgo de fibrilación auricular a través del control de la presión arterial, lo cual hace pensar que el control de la hipertensión no ejerce un papel trascendental en la prevención de la fibrilación auricular en individuos sin enfermedad cardiovascular establecida ${ }^{23}$; sin embargo sí cumple uno fundamental en la prevención del riesgo tromboembólico como se demostró en el estudio LIFE con losartán ${ }^{24,25}$.

Está bien establecido y documentado que la hipertensión arterial se asocia con mayor riesgo de fibrilación auricular y sus complicaciones, particularmente ataque cerebrovascular. Aunque el tratamiento de la hipertensión no ha demostrado claramente una disminución en la incidencia de fibrilación auricular, es un componente importante en la reducción del riesgo cardioembólico y de cualquier estrategia de manejo de fibrilación auricular. Se recomienda el uso de IECA o ARA ॥ como razonable para prevención primaria de fibrilación auricular en el contexto de pacientes con falla cardíaca y disfunción ventricular izquierda (clase IIA, nivel B). Para prevención primaria en hipertensión arterial sin falla cardíaca, se recomienda como IIB, nivel $B$ de evidencia y clase III en pacientes sin enfermedad cardiovascular.

\section{Colesterol}

Los estudios que relacionan la dislipidemia y la incidencia de fibrilación auricular, reportan resultados variables. Algunos han demostrado que los niveles bajos ( $<35 \mathrm{mg} / \mathrm{dl}$ ) de lipoproteína de alta densidad (HDL) se asocian con mayor riesgo de fibrilación auricular de novo ${ }^{26,27}$. Sin embargo, otros no han encontrado asociación entre bajos niveles de HDL y fibrilación auricular, mas aún, altos niveles de colesterol y LDL asociados con bajas tasas de fibrilación auricular ${ }^{28,29}$.

De igual forma, numerosos estudios han evaluado el rol de las estatinas y de los aceites de pescado en la prevención de fibrilación auricular. En un metaanálisis de seis ensayos aleatorizados y controlados con 3.557 pacientes, la terapia con estatinas disminuyó el riesgo relativo de fibrilación auricular en un $61 \%$ cuando se comparó con el manejo convencional $^{30}$. En un análisis de subgrupo, el beneficio se limitó a aquellos con historia previa de fibrilación auricular y aquellos con cirugía cardíaca o manejo para síndromes coronarios agudos.

En cuanto a los aceites de pescado, son limitados los datos de estudios prospectivos acerca del papel de estos en la disminución de la incidencia de fibrilación auricular. Dos estudios aleatorizados doble ciego controlados con placebo de ácidos omega 3 (4-8 g/d), no lograron demostrar una reducción en la recurrencia de fibrilación auricular en pacientes con antecedente de fibrilación auricular paroxística o persistente. En un metaanálisis el suplemento con aceites de pescado no se asoció con una reducción significativa del riesgo de fibrilación auricular ${ }^{31-33}$.

El uso de estatinas, sin embargo, se ha asociado con una disminución del riesgo de fibrilación auricular postoperatoria. Un meta-análisis de estudios aleatorizados controlados y estudios observacionales en cirugía cardíaca con 17.643 pacientes, demostró menor incidencia de fibrilación auricular con el uso prequirúrgico de estatinas ${ }^{34}$.

En general, los resultados que relacionan la dislipidemia y la incidencia de fibrilación auricular, son mixtos, así como lo son el uso de estatinas y aceites de pescado en la prevención. Con base en la evidencia, la recomendación del uso de agentes hipolipemiantes con el propósito de prevenir la fibrilación auricular o su manejo, se limita a su papel en el contexto de cirugía cardíaca.

Se recomienda como estrategia de prevención primaria en fibrilación auricular, el uso de estatinas en el contexto de postquirúrgico de cirugía cardíaca (clase IIB, nivel de evidencia A).

\section{Diabetes}

Es un factor de riesgo independiente para fibrilación auricular $^{35}$, lo cual ha sido corroborado con estudios de cohortes prospectivos a largo plazo. En el Framingham, la diabetes se asoció con un riesgo $40 \%$ y $60 \%$ mayor de fibrilación auricular en hombres y en mujeres, respectivamente, después de 38 años de seguimiento.

Acerca de los mecanismos fisiopatológicos subyacentes entre la asociación de diabetes y fibrilación auricular, se ha implicado a la neuropatía autonómica cardíaca que conlleva sobreactividad simpática y remodelamiento neurológico ${ }^{36}$. La disfunción de la actividad autonómica cardíaca puede 
desencadenar fibrilación auricular, especialmente en circunstancias de cambios en el tono vagal ${ }^{37}$.

De otro lado, hay limitación en los resultados del manejo de la diabetes y el riesgo de fibrilación auricular. El estudio "Action to control Cardiovascular Risk in Diabetes" aleatorizó 10.251 pacientes a un control glicémico intensivo (HbA1c $<6 \%$ ) vs. objetivo convencional de 7-7,9\% de HbA1c. No se apreció diferencia en fibrilación auricular de novo entre los dos brazos ${ }^{38}$.

En resumen, al revisar la literatura que relaciona el control de la diabetes y la fibrilación auricular, la evidencia no es suficiente para recomendar algún manejo de la diabetes en particular para prevenir el riesgo de desarrollar fibrilación auricular. Sin embargo, es razonable la hipótesis que el manejo óptimo de la diabetes y la prevención de sus complicaciones cardiovasculares, puede reducir indirectamente dicho riesgo ${ }^{39}$.

\section{Síndrome de apnea-hipopnea obstructiva del sueño}

Es una condición altamente prevalente en pacientes con fibrilación auricular. En un análisis prospectivo, aproximadamente el $50 \%$ de los pacientes con fibrilación auricular presentaron dicho síndrome vs. un $32 \%$ en el grupo control ${ }^{37}$. Después de un análisis multivariado en el que se evaluaron los factores de riesgo tradicionales para este síndrome, la fibrilación auricular presentó mayor asociación que otros como IMC, hipertensión arterial o diabetes.

Los mecanismos por los cuales el síndrome de apneahipopnea obstructiva del sueño (SAHOS) contribuye al riesgo de fibrilación auricular, incluyen: hipoxemia e hipercapnia intermitentes, picos de hipertonía simpática e hipertensión durante momentos de apnea y aumento de fenómenos inflamatorios asociados. Todos estos factores contribuyen pues al remodelamiento de la aurícula izquierda, dilatación y perpetuación de la arritmia ${ }^{40}$.

Igualmente, los estudios que relacionan el manejo correcto del SAHOS con dispositivos de presión continua en la vía aérea (CPAP, su sigla en inglés) en pacientes sometidos a ablación de fibrilación auricular, demuestran menor tasa de recurrencia de crisis en el grupo de pacientes tratados con CPAP que aquellos sin adecuado control del SAHOS.

De igual forma, existe evidencia clínica clara que demuestra que el SAHOS es un factor de riesgo significativo para fibrilación auricular. Adicionalmente, se comprueba la importancia del manejo del SAHOS en el tratamiento de los pacientes con fibrilación auricular, especialmente en aquellos sometido a cardioversión o a ablación.

El peso de la evidencia clínica, sugiere considerar el tamizaje rutinario del SAHOS antes de cualquier estrategia de control del ritmo.

\section{Cigarrillo y alcohol}

Los estudios que asocian el uso de tabaco y el riesgo de fibrilación auricular son conflictivos ${ }^{41-43}$.

El consumo de alcohol se ha asociado con mayor riesgo de fibrilación auricular de manera dependiente de la dosis ${ }^{44}$. En un estudio prospectivo y metaanálisis que analizó el papel de la ingesta leve a moderada de alcohol en el riesgo de

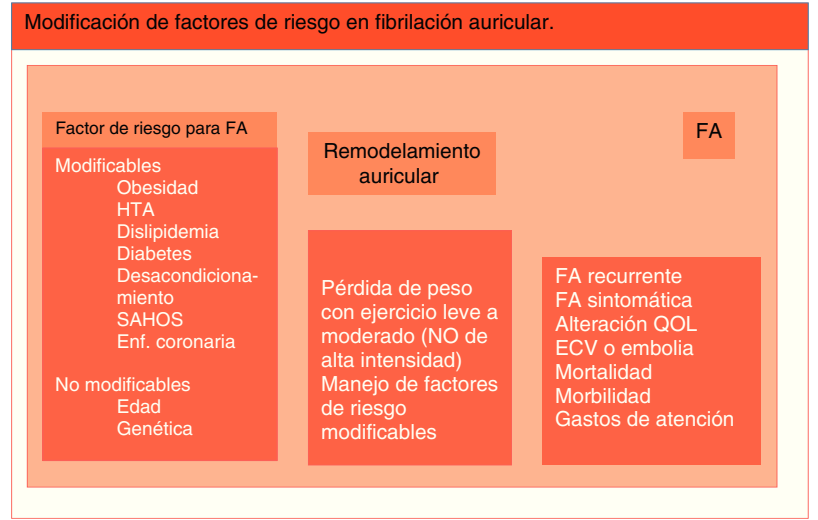

Figura 1 Modificación de factores de riesgo en fibrilación auricular.

Modificada de: Miller JD et al. J Am Coll Cardiol. 2015;66(25):2899-906.

fibrilación auricular e incluyó 79.019 pacientes libres de fibrilación auricular al inicio del estudio, se encontró que el consumo de alcohol, aún a ingestas moderadas (1-3 tragos/día), es un factor de riesgo para fibrilación auricular. Encontraron en general, un $8 \%$ más de riesgo por cada trago diario de ingesta. Este estudio concluyó que el consumo de alcohol está relacionado de manera positiva con el riesgo de fibrilación auricular. Aún el consumo moderado, que disminuye el riesgo de otras enfermedades cardiovasculares, parece aumentar ligeramente el riesgo de fibrilación auricular.

Aunque el consumo de tabaco y alcohol se ha relacionado con mayor riesgo de sufrir fibrilación auricular, el efecto de suspender el consumo de tabaco o disminuir el consumo de alcohol en el manejo preventivo de la fibrilación auricular, no es claro. Son estos elementos, sin embargo, componentes de una estrategia razonable para disminuir el riesgo de padecer fibrilación auricular, pero no es posible emitir una recomendación específicamente para mejorar los desenlaces en cuanto a fibrilación auricular.

Los factores de riesgo cardiometabólicos contribuyen al desarrollo y consecuencias de la fibrilación auricular y pueden ser modificados mediante control de peso, ejercicio y manejo de comorbilidades ${ }^{45}$ (fig. 1).

\section{Futuro en la prevención}

La fibrilación auricular se relaciona con procesos fibróticos que pueden preceder la aparición clínica de la arritmia, y a su vez conducen a la progresión del remodelamiento auricular. El tratamiento con inhibidores de la enzima convertidora de angiotensina(IECA), un potente activador del estímulo de las vías fibróticas, así como el uso de bloqueadores del receptor (ARA II) y antagonistas de receptor mineralocorticoides, considera una disminución teórica del proceso fibrótico, sin embargo no ha sido posible comprobarlo en ensayos clínicos. La inhibición del eje R-A-A se recomienda solo en el manejo de la fibrilación auricular cuando la arritmia se asocia con otras condiciones subyacentes coligadas también al remodelamiento fibrótico, tales como hipertensión arterial, hipertrofia ventricular izquierda y disfunción sistólica del ventrículo izquierdo ${ }^{46}$. 
Otros componentes de las vías profibróticas cardíacas (ej.: TGF-B1, PDGF) son blancos terapéuticos atractivos. Su bloqueo ya sea, con anticuerpos bloqueantes u oligonucleótidos de interferencia de vías fibróticas, constituye una estrategia terapéutica, que debe ser probada en ensayos clínicos futuros.

De igual forma, a futuro es promisoria la inhibición de algunas vías de estrés oxidativo relacionado con especies como NADPH, mieloperoxidasa y sintatasa de óxido nítrico, con ácido ascórbico o apocinina, de manera que se logre disminuir la sobreexpresión de estas moléculas y así el remodelamiento estructural y funcional que conllevan ${ }^{47}$.

\section{Inhibición de otras vías inflamatorias}

El factor transformador de crecimiento B1 (TGF-B1) ha demostrado sus propiedades para estimular la actividad fibroblástica y la consecuente conversión fenotípica a miofibroblastos, lo cual es más prominente en la aurícula que en los ventrículos, según modelos caninos. Surge así el tranilast como agente inhibidor del TGF-B1 en sus efectos antirremodelamiento para prevención primaria de la fibrilación auricular en un modelo canino con fibrilación auricular inducida por sobrestimulación ${ }^{48}$.

\section{Estimación del riesgo: puntajes de riesgo}

En un intento por estimar el riesgo de un individuo dado de padecer fibrilación auricular, se han diseñado puntajes de riesgo a 10 años, los cuales valoran con base en predictores ya validados de riesgo según la cohorte de estudio, por ejemplo la de Framingham ${ }^{47}$. El puntaje considera el riesgo a 10 años para individuos entre 45-90 años, hombres y mujeres mediante interacción de variables edad, sexo, IMC, TAS, tratamiento de hipertensión arterial, intervalo PR y presencia de soplo cardíaco significativo y falla cardiaca (puede ampliar información en: www. framinghamheartstudy.org/ risk-functions/atrialfibrillation/10-year-risk.php\#).

Existe, sin embargo, un modelo de predicción a cinco años de fibrilación auricular, el CHARGE-AF Risk Score, construido a partir de cohortes como la del estudio ARIC (Atheroesclerosis Risk in Communities), CHS (Cardiovascular Health Study) y FHS (Framingham Health Study), el cual involucra en el análisis variables étnicas más representativas como la hispana, blanca no hispana y afroamericana y ya ha sido validado a partir de la cohorte de Framingham ${ }^{49}$.

\section{Conclusión}

A pesar de los avances hechos en el tratamiento de la fibrilación auricular en términos de desarrollo tecnológico para la ablación, las estrategias de prevención primaria para controlarla parecen relegadas. La complejidad de interacciones entre variables genéticas, clínicas, celulares, unas modificables o no, hace pensar en la dificultad de encontrar caminos esperanzadores. Es claro creer que el enfoque programático en el control de factores de riesgo como la obesidad, la apnea del sueño y el uso de algunos agentes farmacológicos ampliamente estudiados como la inhibición del sistema R-A-A, pueden dar resultado. La intervención primaria en fibrilación auricular postoperatoria con betabloqueantes prequirúrgicos o amiodarona en pacientes de alto riesgo o estatinas en el postquirúrgico, está mejor cimentada.

\section{Conflicto de intereses}

Los autores declaran no tener ningún conflicto de intereses.

\section{Bibliografía}

1. Rahman F, Kwan GF, Benjamin EJ. Global epidemiology of atrial fibrillation. Nat Rev Cardiol. 2014;11:639-54.

2. Ogden CL, Carroll MD, Kit BK, et al. Prevalence of childhood and adult obesity in the United States, 2011-2012. JAMA. 2014;311:806-14.

3. Huxley RR, Lopez FL, Folsom AR, et al. Absolute and attributable risks of atrial fibrillation in relation to optimal and borderline risk factors: the Atherosclerosis Risk in Communities (ARIC) study. Circulation. 2011;123:1501-8.

4. Tedrow UB, Conen D, Ridker PM, et al. The long- and shortterm impact of elevated body mass index on the risk of new atrial fibrillation: the WHS (Women's Health Study). J Am Coll Cardiol. 2010;55:2319-27.

5. Azarbal F, Stefanick ML, Salmoirago- Blotcher E, et al. Obesity, physical activity, and their interaction in incident atrial fibrillation in postmenopausal women. J Am Heart Assoc. 2014;3:e001127.

6. Karasoy D, Bo Jensen T, Hansen ML, et al. Obesity is a risk factor for atrial fibrillation among fertile young women: a nationwide cohort study. Europace. 2013;15:781-6.

7. Wong $C X$, Sullivan T, Sun MT, et al. Obesity and the risk of incident, post-operative, and post- ablation atrial fibrillation: a meta-analysis of 626, 603 individuals in 51 studies. J Am Coll Cardiol. 2015;1:139-52

8. Frost L, Benjamin EJ, Fenger-Grøn M, et al. Body fat, body fat distribution, lean body mass and atrial fibrillation and flutter. A Danish cohort study. Obesity (Silver Spring). 2014;22:1546-52.

9. Stritzke J, Markus MRP, Duderstadt S, et al. The aging process of the heart: obesity is the main risk factor for left atrial enlargement during aging: the MONICA/KORA (Monitoring of Trends and Determinations in Cardiovascular Disease/Cooperative Research in the Region of Augsburg) study. J Am Coll Cardiol. 2009;54:1982-9.

10. Marcus GM, Olgin JE, Whooley M, et al. Racial differences in atrial fibrillation prevalence and left atrial size. Am J Med. 2010;123, 375.e1-e7.

11. Wong CX, Abed HS, Molaee P, et al. Pericardial fat is associated with atrial fibrillation severity and ablation outcome. J Am Coll Cardiol. 2011;57:1745-51.

12. Balcioglu AS, Cicek D, Akinci S, et al. Arrhythmogenic evidence for epicardial adipose tissue: heart rate variability and turbulence are influenced by epicardial fat thickness. Pacing Clin Electrophysiol. 2015;38:99-106.

13. Stojanovska J, Kazerooni EA, Sinno M, et al. Increased epicardial fat is independently associated with the presence and chronicity of atrial fibrillation and radiofrequency ablation outcome. Eur Radiol. 2015;25:2298-309.

14. Yang $\mathrm{H}$, Youm $\mathrm{YH}$, Vandanmagsar $\mathrm{B}$, et al. Obesity increases the production of proinflammatory mediators from adipose tissue $T$ cells and compromises TCR repertoire diversity: impli- cations for systemic inflammation and insulin resistance. J Immunol. 2010;185:1836-45.

15. Abed HS, Wittert GA, Leong DP, et al. Effect of weight reduction and cardiometabolic risk factor management on symptom 
burden and severity in patients with atrial fibrillation: a randomized clinical trial. JAMA. 2013;310:2050-60.

16. Pathak RK, Middeldorp ME, Meredith M, et al. Long-Term Effect of Goal-Directed Weight Management in an Atrial Fibrillation Cohort: A Long-Term Follow-Up Study (LEGACY). J Am Coll Cardiol. 2015;65:2159-69.

17. Abed HS, Nelson AJ, Richardson JD, et al. Impact of weight reduction on pericardial adipose tissue and cardiac structure in patients with atrial fibrillation. Am Heart J. 2015;169, 65562.e2.

18. Mozaffarian D, Furberg CD, Psaty BM, et al. Physical activity and incidence of atrial fibrillation in older adults: the Cardiovascular Health Study. Circulation. 2008;118:800-7.

19. Pathak RK, Elliott A, Middeldorp ME, et al. Impact of CARDIOrespiratory FITness on Arrhythmia Recurrence in Obese Individuals with Atrial Fibrillation: the CARDIO-FIT study. J Am Coll Cardiol. 2015;66:985-96.

20. Brunner KJ, Bunch TJ, Mullin CM, et al. Clinical predictors of risk for atrial fibrillation: implications for diagnosis and monitoring. Mayo Clin Proc. 2014;89:1498-505.

21. Vaziri SM, Larson MG, Lauer MS, et al. Influence of blood pressure on left atrial size. The Framingham Heart Study. Hypertension. 1995;25:1155-60.

22. Kottkamp H. Human atrial fibrillation substrate: towards a specific fibrotic atrial cardiomyopathy. Eur Heart J. 2013;34:2731-8.

23. January CT, Wann LS, Alpert JS, et al. 2014 AHA/ACC/HRS guideline for the management of patients with atrial fibrillation: a report of the American College of Cardiology/American Heart Association Task Force on Practice Guidelines and the Heart Rhythm Society. J Am Coll Cardiol. 2014;64:e1-76.

24. Lip GY, Nieuwlaat R, Pisters R, et al. Refining clinical risk stratification for predicting stroke and thromboembolism in atrial fibrillation using a novel risk factor-based approach: the Euro Heart Survey on Atrial Fibrillation. Chest. 2010;137:263-72.

25. Wachtell K, Lehto M, Gerdts E, et al. Angiotensin II receptor blockade reduces new-onset atrial fibrillation and subsequent stroke compared to atenolol: the Losartan Intervention For End Point Reduction in Hypertension (LIFE) study. J Am Coll Cardiol. 2005;45:712-9.

26. Haywood LJ, Ford CE, Crow RS, et al. ALLHAT Collaborative Research Group. Atrial fibrillation at baseline and during follow-up in ALLHAT (Anti- hypertensive and Lipid-Lowering Treatment to Prevent Heart Attack Trial). J Am Coll Cardiol. 2009;54:2023-31.

27. Alonso A, Yin X, Roetker NS, et al. Blood lipids and the incidence of atrial fibrillation: the Multi- Ethnic Study of Atherosclerosis and the Framing- ham Heart Study. J Am Heart Assoc. 2014;3:e001211.

28. Lopez FL, Agarwal SK, Maclehose RF, et al. Blood lipid levels, lipid-lowering medications, and the incidence of atrial fibrillation: the atherosclerosis risk in communities study. Circ Arrhythm Electrophysiol. 2012;5:155-62.

29. Psaty BM, Manolio TA, Kuller LH, et al. Incidence of and risk factors for atrial fibrillation in older adults. Circulation. 1997;96:2455-61.

30. Fauchier L, Pierre B, de Labriolle A, et al. Antiarrhythmic effect of statin therapy and atrial fibrillation a meta-analysis of randomized controlled trials. J Am Coll Cardiol. 2008;51:828-35.

31. Nigam A, Talajic M, Roy D, et al. AFFORD Investigators. Fish oil for the reduction of atrial fibrillation recurrence, inflammation, and oxidative stress. J Am Coll Cardiol. 2014;64:1441-8.
32. Kowey PR, Reiffel JA, Ellenbogen KA, et al. Efficacy and safety of prescription omega-3 fatty acids for the prevention of recurrent symptomatic atrial fibrillation: a randomized controlled trial. JAMA. 2010;304:2363-72.

33. Liu T, Korantzopoulos P, Shehata M, et al. Prevention of atrial fibrillation with omega-3 fatty acids: a meta-analysis of randomised clinical trials. Heart. 2011;97:1034-40.

34. Liakopoulos OJ, Choi YH, Kuhn EW, et al. Statins for prevention of atrial fibrillation after cardiac surgery: a systematic literature review. J Thorac Cardiovasc Surg. 2009;138, 678-86.e1.

35. Huxley RR, Filion KB, Konety S, et al. Meta-analysis of cohort and case-control studies of type 2 diabetes mellitus and risk of atrial fibrillation. Am J Cardiol. 2011;108:56-62.

36. Tesfaye S, Chaturvedi N, Eaton SE, et al. EURODIAB Prospective Complications Study Grloup. Vascular risk factors and diabetic neuropathy. N Engl J Med. 2005;352:341-50.

37. Dimmer C, Tavernier R, Gjorgov N, et al. Variations of autonomic tone preceding onset of atrial fibrillation after coronary artery bypass grafting. Am J Cardiol. 1998;82:22-5.

38. Fatemi O, Yuriditsky E, Tsioufis C, et al. Impact of intensive glycemic control on the incidence of atrial fibrillation and associated cardiovascular outcomes in patients with type 2 diabetes mellitus (from the Action to Control Cardiovascular Risk in Diabetes Study). Am J Cardiol. 2014;114:1217-22.

39. Miller JD, Aronis KN, Chrispin J, et al. Obesity, exercise, obstructive sleep apnea, and modifiable atherosclerotic cardiovascular disease risk factors in atrial fibrillation. JACC. 2015;66:2899-906.

40. Gami AS, Pressman G, Caples SM, et al. Association of atrial fibrillation and obstructive sleep apnea. Circulation. 2004;110:364-7.

41. Somers VK, Dyken ME, Clary MP, et al. Sympathetic neural mechanisms in obstructive sleep apnea. J Clin Invest. 1995;96:1897-904.

42. Krahn AD, Manfreda J, Tate RB, et al. The natural history of atrial fibrillation: incidence, risk factors, and prognosis in the Manitoba Follow-Up Study. Am J Med. 1995;98:476-84.

43. Stewart S, Hart CL, Hole DJ, et al. Population prevalence, incidence, and predictors of atrial fibrillation in the Renfrew/Paisley study. Heart. 2001;86:516-21.

44. Chamberlain AM, Agarwal SK, Folsom AR, et al. Smoking and incidence of atrial fibrillation: results from the Atherosclerosis Risk in Com- munities (ARIC) study. Heart Rhythm. 2011;8:1160-6.

45. Kodama S, Saito K, Tanaka S, et al. Alcohol consumption and risk of atrial fibrillation: a meta-analysis. J Am Coll Cardiol. 2011;57:427-36.

46. Camm AJ, Kirchhof P, Lip GY, et al. for the European Heart Rhythm Association; European Association for Cardio-Thoracic Surgery. Guide- lines for the management of atrial fibrillation: the Task Force for the Management of Atrial Fibrillation of the European Society of Cardiology (ESC). Eur Heart J. 2010;31:2369-429.

47. Violi F, Carnevale R, Calvieri C, et al. Nox2 up-regulation is associated with an enhanced risk of atrial fibrillation in patients with pneumonia. Thorax. 2015;70:961-6.

48. Nakatani $Y$, Nishida K, Sakabe M, et al. Transilast and atrial fibrillation. J Am Coll Cardiol. 2013;61:582-8.

49. Schnabel RB, Sullivan LM, Levy D, et al. Development of a risk score for atrial fibrilation: A community-based cohort study. Lancet. 2009;373:739-45. 